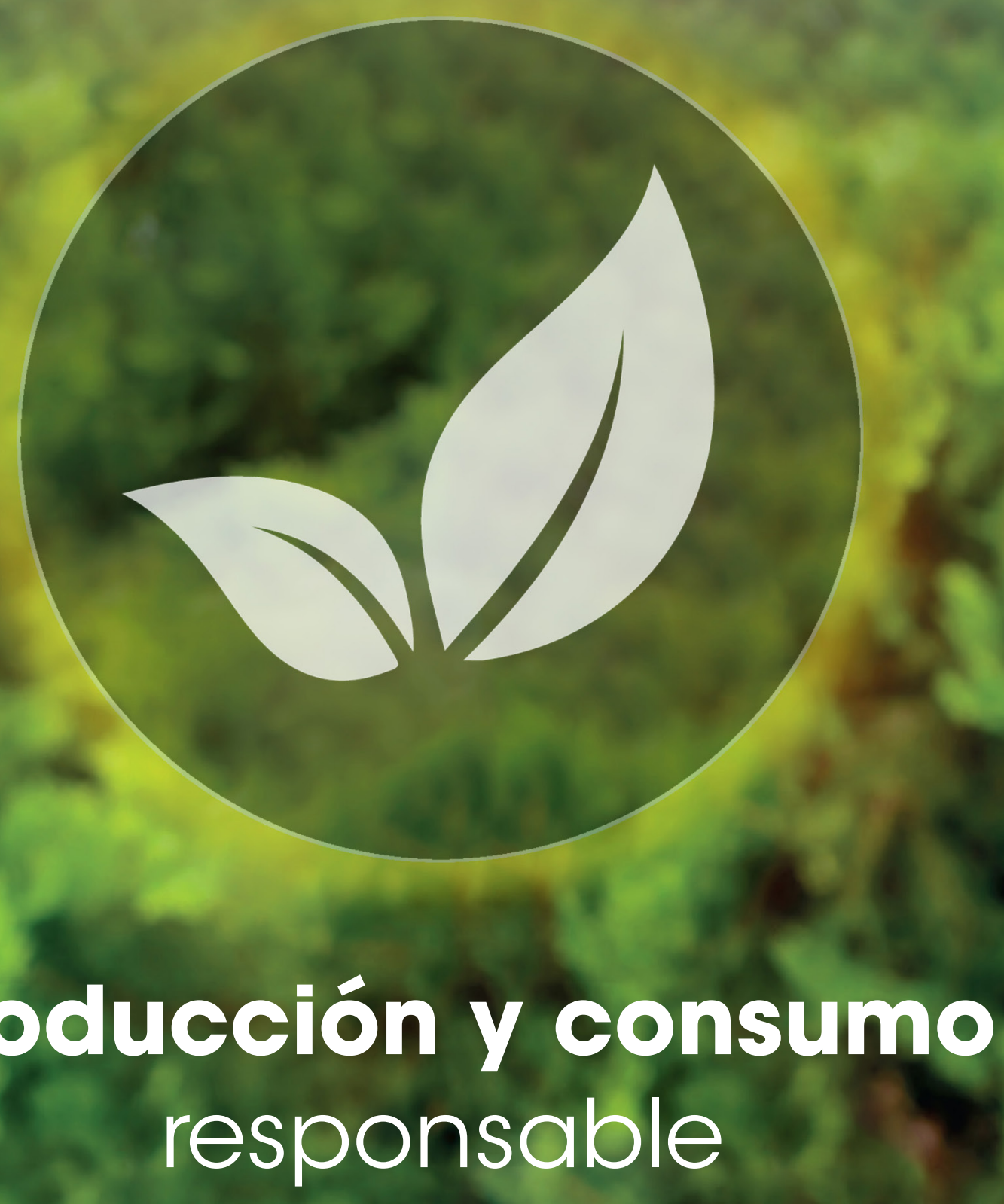




\section{ANTENNA FOR THE ACQUISITION OF METEOROLOGICAL SATELLITE SIGNALS (ASSM)}

Edwin Fernando Pineda Vargas ${ }^{16}$

Fundación Universitaria San Mateo

\section{Resumen:}

Actualmente el mundo se encuentra en una crisis debido al cambio climático. La reciente ola de desastres naturales, producto del aumento de la temperatura global, ha desencadenado que los desastres naturales aumenten su poder de destrucción. Si es posible detectar estos fenómenos antes de tiempo, y se pueden predecir, se puede preparar mejor nuestros lugares de vivienda y así resguardarse de las calamidades climáticas. Por ello, el ser humano ha implementado una serie de medidas que involucran la comunicación mediante satélites geoestacionarios; capturan datos de la atmósfera como fotografías y medidas de variables meteorológicas para establecer los posibles efectos de las variaciones climáticas en las diferentes zonas del planeta. Según el diseño y la fabricación de una antena que capte la señal del satélite de dominio público, enviada por los satélites NOAA 15, 18 y 19, se espera capturar datos sobre las condiciones climáticas de Bogotá; esta señal contiene imágenes climatológicas del área sobrevolada por el satélite, en este caso la ciudad de Bogotá.

\section{Palabras clave:}

Antena, meteorología, geoestacionario, NOAA, redes satelitales. 


\begin{abstract}
:
The world is currently in a several crises due to climate change. The recent wave of natural disasters, due to the rise in global temperature, has triggered that natural disasters increase their destruction power. If it is possible to detect these phenomena ahead of time, and it can be predicted, we can better prepare the housing places and thus protect ourselves from climatic calamities. Therefore, the human being has implemented a series of measures where communication is involved through geostationary satellites. Where atmospheric data are captured, such as photographs and measurements of meteorological variables, the potential effects of climatic variations in the different areas of the planet must be established. Through the design and manufacture of an antenna, that allows to capture the signal from the public domain satellite (ATP) sent by the NOAA satellites 15, 18 and 19, it is expected to capture data on the Bogotá climatic conditions, since this signal contains climatological images of the area overflew by the satellite, in this case, in the city of Bogotá.
\end{abstract}

\title{
Keywords:
}

Antenna, geostationary, meteorology, NOAA, satellite networks.

\section{Introducción}

En el constante crecimiento del desarrollo tecnológico e intelectual de las naciones, el mundo científico se ha enfocado en la caracterización de diversas variables físicas. Una de ellas es la temperatura; ha brindado información relevante en el proceso del movimiento molecular de las partículas de materia que están expuestas a ella. Así, brinda respuestas a fenómenos atmosféricos como las lluvias torrenciales, los huracanes, los tornados, entre otros fenómenos atmosféricos. 
Sin embargo, los fenómenos previos se relacionan entre sí. Una vez analizado uno (1) de ellos, es imposible determinar las variaciones climáticas en una sola área. Por tal razón, se han implementado modelos estocásticos que permiten establecer la proyección de estas variables, su correlación y los posibles efectos que causan en un determinado tiempo y área geográfica. En este sentido, la humanidad se ha enfocado en lograr la caracterización del clima para poder establecer los efectos de grandes fenómenos naturales como lo son los huracanes, tornados, lluvias torrenciales, sequías, entre otros.

Ahora bien, para lograrlo no es suficiente establecer una estación meteorológica en la tierra como base. Por lo anterior, se han enviado satélites a la órbita geoestacionaria de la tierra para desde allí poder tomar una serie de fotografías que permitan a los meteorólogos establecer el comportamiento de los fenómenos climatológicos con mayor facilidad. Ahora bien, la implementación de los satélites logra fotografías de la tierra desde la órbita geoestacionaria. El envío de la información es realizado a través de señales moduladas en frecuencia, o en fase, mediante técnicas como FM, XFSK, QAM, en cada una de sus variantes. Esto, a partir de los bits que logran trabajar, para establecer un correcto envío de la comunicación.

Dicho envío de información normalmente está establecido por el fabricante del satélite, según los estándares planteados para su envío. De allí, el reto se convierte en recibir la información, decodificarla y establecer lo que sucede visualmente. La presente investigación se enfoca en un proyecto a largo plazo; busca capturar las señales enviadas por el grupo de satélites National Oceanic and Atmospheric Administration (NOAA) 15, 18 y 19, de dominio público (ATP). Es decir, la información se puede acceder libremente por cualquier persona que se encuentre en la trayectoria del satélite. Lo anterior es planteado, realizado y ejecutado por el Semillero de investigación en Redes Satelitales de la Fundación Universitaria San Mateo. 


\section{Conceptos generales sobre satélites}

Para poder desarrollar este proyecto, es necesario conocer una serie de definiciones fundamentales que permiten la captura de las imágenes provenientes de los satélites NOAA 15, 18 y 19.

\section{¿Qué es un satélite?}

Un satélite es un dispositivo de comunicaciones cuya principal función radica en recibir, amplificar y trasladar en frecuencia señales de radio; enviadas a estaciones receptoras de destino, de manera simultánea. Las estaciones pueden ser terrestres fijas, móviles, o estar en la órbita geoestacionaria de manera fija o móvil [1][2]. Cuando se trata de la banda C de transmisión, normalmente el oscilador de radiofrecuencia, ubicado al interior del satélite, está configurado a 2225 megahercios (MHz) [3][4]. Básicamente, el satélite está formado por:

- Antena Receptora: Es la encargada de recibir las señales de radiofrecuencia provenientes del transmisor, ubicado en el emisor de la señal [5].

- Amplificador de RF: Amplifica las señales de radiofrecuencia recibidas por la antena receptora con el fin de ofrecer una señal adecuada y realizar su correspondiente tratamiento y filtrado para ser retransmitida [2][6]. En el caso de la última etapa, cuando el amplificador se encuentra antes de la antena transmisora, se debe tener en cuenta la potencia de salida de la señal; debe ser tan alta que logre propagarse a través del medio y llegar al receptor de interés. Cuando se tienen estos amplificadores en las últimas etapas de retransmisión de la señal, es necesario garantizar una potencia de salida que permita a la señal atravesar el interfaz vacío - aire y logre llegar al receptor, ubicado en la capa terrestre, con la potencia adecuada [7][3].

Cabe aclarar que el mismo concepto es aplicado cuando la comunicación se da entre dos (2) satélites ubicados en el espacio. No obstante, la potencia es menor; el medio de transmisión es el vacío, el cual es homogéneo y no presenta pérdidas por dispersión de la onda electromagnética. Aunque la permitividad 
del aire, y la del vacío, es 8.8541878176×10-12 C2 / (N.m2) [2][8][9], el problema de la pérdida de potencia de la onda electromagnética proviene de los fenómenos de dispersión de la onda en el aire, compuesto de diversos materiales gaseosos como el oxígeno, nitrógeno e hidrógeno principalmente.

Los anteriores materiales, cuando son sometidos a diversos cambios energéticos, pueden formar nuevas moléculas que originan cambios de permitividad en el medio. Por ejemplo, el agua; cuando los átomos de oxígeno e hidrógeno se someten a las condiciones electrónicas, de presión y temperatura adecuadas, se forman moléculas de agua. Por su composición química, el agua tiene una permitividad eléctrica cercana a 80, que altera todas las propiedades físicas de transmisión normal de una onda electromagnética [1][2][3][8].

- Oscilador Local: Funciona como un sistema de referencia para mezclar la señal recibida y así realizar un promedio para hallar la frecuencia intermedia (FI) entre la señal generada por el oscilador y la señal recibida por la antena, amplificada con anterioridad. Algunas frecuencias de operación de los satélites, con sus estaciones terrestres, son de $3700 \mathrm{MHz}-4200 \mathrm{MHz}$ (cuando la comunicación se establece del satélite a la estación terrestre) y de 5925 $\mathrm{MHz}-6425 \mathrm{MHz}$ (cuando la comunicación es de la estación terrestre al satélite) [6][8].

- Mezclador de Señales de Radiofrecuencia: Este dispositivo se encarga de tomar la señal proveniente del amplificador de radio frecuencia y mezclarla con la señal del oscilador local. De allí se obtiene al finalizar la suma de ambas señales y se puede establecer una frecuencia intermedia de la señal recibida [9] [10].

- Antena Transmisora: Toma la señal proveniente del último amplificador y la envía al medio que propaga la señal para ser recibida por la estación base, o receptor, donde se desea enviar la señal. En este caso, el primer medio es el vacío y el segundo es la atmósfera terrestre [7]. 
Figura 1. Diagrama de bloques del funcionamiento de un satélite.

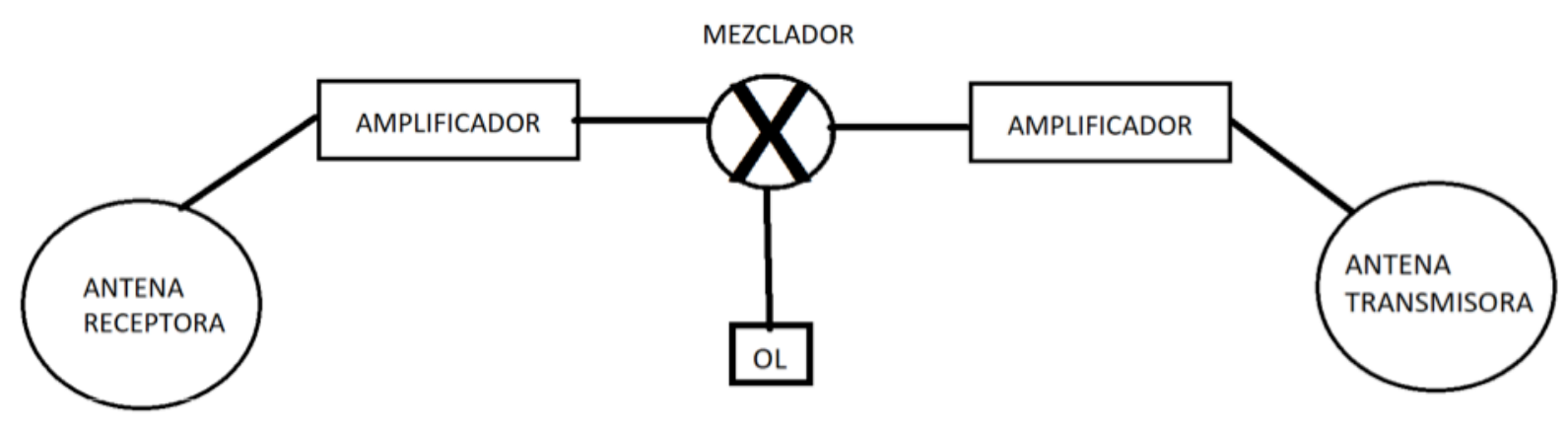

Fuente: elaboración propia.

Así, en la figura 1 se aprecia el esquema básico de comunicaciones de un satélite en diagrama de bloques [1][3].

Como el satélite se encuentra sometido a una serie de fuerzas, como la gravitatoria de la tierra y las fuerzas centrífugas provenientes de otros cuerpos celestes, el satélite debe estar en constante corrección de su posición. Esto, principalmente, por la variación de los campos electromagnéticos a los cuales es sometido el mismo en la órbita geoestacionaria. Por lo anterior, es necesario contar con un control de posición; permite establecer una posición de equilibrio en el mismo, corrigiendo sus desviaciones, producto de las fuerzas anteriormente mencionadas. Dicha posición es corregida mediante la expulsión de combustible sólido; aprovecha la tercera ley de Newton para poder estabilizar el satélite, según sea el factor de corrección a implementar. Así, logra estabilizar el satélite orbitalmente y lo posiciona en un solo sitio [1].

Además, los satélites se alimentan con fuentes de energía solar normalmente, ideales para este tipo de dispositivos pues brindan autonomía energética al mismo. Las fuentes de energía solar están compuestas por paneles solares y convertidores DC-DC de tipo BUCK y BOOST; correspondientes a tipologías elementales para este tipo de sistemas. Adicional a estos, pueden im- 
plementarse fuentes combinando las dos (2) topologías anteriores, al igual que una topología Full Bridge, variación del convertidor Buck [1].

Respecto a su forma de emisión, se establece un patrón denominado "huella satelital"; corresponde al patrón de radiación de la antena que hace parte del sistema de transmisión del satélite. Por lo anterior, se tiene en cuenta cuatro (4) tipos de haces de radiación en las antenas satelitales [1].

a. Haz global: Permite que el satélite radie o ilumine una tercera parte del globo terráqueo, de tal forma que logre comunicar a toda la tierra con tan solo tres (3) satélites.

b. Haz hemisférico: Concentra su potencia en radiar una amplia región de la tierra, como puede ser un continente.

c. Haz regional: Estos permiten que la mayor cantidad de potencia se concentre en pequeñas zonas o países determinados.

d. Haz spot: Concentran la mayor cantidad de potencia en una región, o zona mínima, pequeña, al interior de un país [1].

El uso de los haces regionales y spot han permitido reducir el costo de operación de sistemas satelitales; brinda la posibilidad de diseñar y establecer pequeñas estaciones para aplicaciones, disminuyendo el costo terrestre. Por otro lado, respecto a la polarización de las antenas conectadas al satélite; de acuerdo a la forma en que reciben las ondas electromagnéticas, existen dos (2) tipos: lineal, cuando es vertical u horizontal, y circular, cuando es derecha o izquierda [7] [8]. Los satélites también se pueden clasificar según el servicio para el cual son diseñados, de uso internacional y doméstico [5].

En cuanto a la referencia de los satélites de uso internacional, son aquellos que tienen haces globales y hemisféricos para cubrir comunicaciones en todo el mundo; a su vez adicionan haces regionales y spots sobre zonas específicas, o de gran interés para quien diseña el satélite. Los primeros satélites de este tipo fueron los de la serie Intelsat; tenían spots concentrados sobre zonas de 
América del sur y América del Norte, posibilitando el uso de satélites en países en vías de desarrollo en esa zona (como es el caso de Colombia) pues los sistemas de recepción son económicos. Por lo anterior, los altos valores de Potencia Isotrópica Radiada Equivalente (PIRE), los cuales se encuentran mayores a 36 Db W, antes eran logrados en potencias entre 23,5 y 26 dB W, según la zona a tratar [1].

Según la frecuencia de operación, los satélites tenían frecuencias centrales con diferentes transpondedores y anchos de banda y posibilitaban el uso en telefonía, televisión (TV) o datos. De acuerdo a las necesidades del cliente, las frecuencias centrales de los transpondedores se utilizan para la retransmisión de señales de TV principalmente, de tal forma que se logre ubicar el transpondedor completo o en medio, para el caso concreto del satélite Intelsat. Sin embargo, varía según la aplicación y el satélite a implementar. En cuanto a la polarización, es posible utilizar varias frecuencias, a partir de la implementación de varias polarizaciones al momento de realizar el envío de la onda electromagnética al espacio [7].

Si se llegase a dar el caso de transmitir dos (2) señales de TV en el mismo haz, pero con polarizaciones contrarias, las antenas receptoras deben contar con alto rechazo a la polarización cruzada, de tal forma que permita captar únicamente la señal de interés. Para no alterar, y desmejorar la calidad de la señal en la recepción, es necesario tener en cuenta que la potencia máxima debe ser superior a 25 decibelios (Db). La mayoría de los satélites cuentan con polarización izquierda y transmiten en derecha, tal y como sucede con el satélite Intelsat. No obstante, recientemente se ha logrado instrumentar la posibilidad de que tanto el transmisor como el receptor funcionen en cualquiera de las dos polarizaciones [1].

Cuando la investigación hace referencia a satélites de uso doméstico, se habla de satélites como el Brasilsat, Palcipa, Galaxy, entre otros [1]. Debido a que son de uso doméstico, el haz emitido por la antena del satélite normalmente posee un footprint o una guía, de acuerdo a la forma del país, de tal manera 
cubra al país con la mayor potencia posible. Sin embargo, si el país es muy grande, se pueden cubrir con dos (2) o tres (3) haces para así aumentar la PIRE con la mayor cantidad de zonas a implementar. Empero, respecto a la frecuencia, la central de cada una de las portadoras se separa $20 \mathrm{MHz}$ de las aledañas, para así evitar interferencias entre sí [1]. Por último, en relación con la polarización, a diferencia de los satélites internacionales, los domésticos utilizan polarización lineal, izquierda o derecha repartidas normalmente en pares e impares [1].

\section{Geometría del satélite.}

Los satélites normalmente describen órbitas elípticas o circulares, sin embargo, para efectos de la investigación, es necesario analizar órbitas circulares y ecuatoriales, aquellas que comúnmente utilizan los servicios de comunicaciones telefónicas y de TV. Lo anterior, debido a que los datos transmitidos por el satélite NOAA están en el rango de frecuencia de las señales de TV; la altitud a la cual ocurre esta órbita se determina según el tiempo que tarda el satélite en dar una vuelta a la tierra, en tiempo sidéreo, es decir, un tiempo cercano a 23 horas, 56 minutos y cuatro (4) segundos.

En efecto, las ecuaciones que describen el sistema se observan en las ecuaciones (1) (2) y (3), en donde se aplican las ecuaciones físicas para la atracción de dos cuerpos en condición de equilibrio denotada con la letra F, la fuerza centrífuga denotada con la letra P y las ecuaciones que relacionan la posición de equilibrio para que el satélite se encuentre en órbita respectivamente [1].

$$
F=\frac{\left(m_{s} m_{t}\right)}{d^{2}}
$$

$$
P=\frac{\left(m_{s} v^{2}\right)}{d}
$$




$$
\frac{\left(m_{s} m_{t}\right)}{d^{2}}=\frac{\left(m_{s} v^{2}\right)}{d}
$$

Siendo $d=h+R$.

Por lo tanto:

$$
v^{2}=\frac{m_{t}}{(R+h)}
$$

La circunferencia de la órbita es:

$$
l=2 \pi(R+h)
$$

Y la $v=$ distancia $/$ tiempo $=2 \pi(R+h) /$ ts

Por lo tanto queda:

$$
h=\sqrt{\frac{m_{t}\left(t_{s}\right)^{2}}{(2 \pi)^{2}}}-R
$$


Según lo anterior, h corresponde a la altura del satélite con respecto a la corteza terrestre. R es el radio de la tierra, el cual corresponde a 6375 Km. Mt es la masa gravitacional de la tierra, aproximadamente de $398600 \mathrm{Km} 3$ / seg2 y ts es el tiempo de un día sidéreo[1], anteriormente mencionado. Este se pude calcular como:

$$
h=\left(\frac{398600 \cdot(86164)^{2}}{(2 \pi)^{2}}\right)^{\frac{1}{3}}-6375=35789 K m
$$

La altitud de $35789 \mathrm{Km}$ produce la órbita geoestacionaria; provoca que el satélite se observe como un punto fijo para un observador ubicado en cualquier lugar de la corteza terrestre[1].

\section{Propuesta desde el semillero de investigación en redes satelitales}

En el avance y mejoramiento constante de las habilidades de los estudiantes de la Fundación Universitaria San Mateo, desde la docencia de la institución se plantea el proyecto de diseñar y construir una antena para la recepción de señales de un satélite de dominio público. Dichas señales contienen información sobre imágenes climatológicas del área sobrevolada por el satélite.

\section{Objetivo general.}

Diseñar, simular, construir y probar una antena para la recepción de la señal satelital de dominio público tipo Automatic Picture Transmission (APT) enviada por los satélites NOAA 15, 18 y 19 en la ciudad de Bogotá. 


\section{Objetivos específicos.}

- Desarrollar prototipos simulados de antenas que permitan captar la señal de alguno de los satélites de la red NOAA.

- Implementar algoritmos de captación de ondas electromagnéticas (al igual que la captura de su contenido de información) mediante plataformas de software libre para decodificar la información proveniente de los satélites de la red NOAA.

- Implementar algoritmos computacionales que permitan captar y reproducir la señal de uno de los satélites de la red NOAA para conocer las condiciones climáticas de Bogotá en un determinado instante de tiempo.

- Desarrollar prototipos físicos de antenas que permitan la captura de los datos recibidos por alguno de la red de satélites NOAA.

\section{Especificaciones}

Dentro de la investigación realizada por parte del autor de esta propuesta, para el levantamiento de requerimientos del proyecto, se evidencia la necesidad de contar con las siguientes especificaciones.

- Frecuencia de operación: $137 \mathrm{MHz}$

- Ancho de banda: > 40 Kilohertzios $(\mathrm{KHz})$

- Polarización de la Antena a Diseñar: Circular derecha (RHCP)

- Puerto de conexión del receptor: SMA acoplado a 50 ohmios (ohm).

\section{Resultados}

A la fecha no es posible entregar resultados; el grupo lleva un par de meses desde su creación. En un primer acercamiento, esta investigación ha realizado un trabajo bibliográfico. Para la realización de fases futuras, se espera poder contar con el apoyo financiero, técnico y logístico de la Fundación Universitaria San Mateo. 


\section{Conclusiones}

Hasta el momento, se ha realizado una labor netamente bibliográfica. El tema central fue cómo solucionar la identificación de variables físicas relacionadas con el clima, su cuantización, cuantificación y demás aspectos que permitan realizar una correcta y adecuada caracterización. 


\section{Referencias bibliográficas}

[1] J. A. Bava y A. J. Sanz, Microondas y Recepción Satelital, Buenos Aires, Argentina: Editorial Hispano Americana S.A., 1995.

[2] Ahmet F. Yagli, Mesut Gokten, Lokman Kuzu, Hasan H. Ertok and Senol Gulgonul, An Antenna Array for Ku band Satellite Reception, Turkey: Space Technologies Research Inst., 2016.

[3] Satish kumar Bandlamudi, Puneet Kumar Mishra, Sasikanta Kumar, Haindavi M, Renuka R, S. S. Kumar and V. K. Hariharan, Compact Ultra Wide Band Sinuous Antenna, Satellite Antenna Characterization Test \& Design Section, Electrical Design \& Measurement Division Systems Integration Group, Bangalore, India: ISRO Satellite Centre (ISAC), 2017.

[4] Sasikanta Kumar, Puneet Kumar Mishra, Satish kumar Bandlamudi, Haindavi M, Renuka R, S. S. Kumar and V. K. Hariharan, Experimental and Parametric Studies on Ultra Wide-Band and Low X-Pol Helical Antenna, Electr. Power Syst. Satellite Antenna Characterization Test \& Design Section, Electrical Design \& Measurement Division Systems Integration Group, Bangalore, India: ISRO Satellite Centre, 2017.

[5] Haindavi M, Puneet Kumar Mishra, Sasikanta Kumar, Satish kumar Bandlamudi, Renuka R, S. S. Kumar and Dr.V. K. Hariharan, An Ultra Wide C-Band Compact Range Feed, India: Satellite Antenna Characterization Test \&
Design Section, Electrical Design \& Measurement Division Systems Integration Group, 2018.

[6] Jeom-Hun Lee, J-S Choi, Jaehoon Kim, S-P Lee, Seung-hyeub Oh, "Performance Analysis of Satellite Payload Antenna Design Result", Global Area Wireless Technology Research Group, ETRI Daejon-City, S Korea, 2006.

[7] Shivam Arora, Venkata Sitaraman Puram, Sandhya Reddy B, Senthil kumar V,C Sriharsha and D V Ramana, X-Band Choked Horn Antenna for On-Board TTC Downlink of Deep Space Satellite Applications, Bangalore, India: Communication Systems Group, ISRO Satellite Centre, HAL Airport Road, 2017.

[8] V. Pascale, D. Maiarelli, L. D’Agristina, N. Gatti, Design and Qualification of Ku-Band Radiating Chains for Receive Active Array Antenna of Flexible Telecommunication Satellites, Roma, Italia: Antenna \& RF Passive Dpt., Space Engineering S.p.A., 2019.

[9] S. Rao, Satellite Communication Antennas: Challenges for the Next Generation Payloads, USA: Northrop Grumman, 2015.

[10] R. Yousefian y S. Kamalasadan, Design of quadrifilar helical and spiral antennas in the presence of satellite-mobile handsets using genetic Algorithms, Bradford, UK: Mobile and Satellite Communications Research Centre, University of Bradford, 2009. 